\title{
Generating synthetic rainfall using a disaggregation model
}

\author{
Sherin Ahamed, Julia Piantadosi, Manju Agrawal, John Boland \\ School of Information Technology and Mathematical Sciences, University of South Australia, Mawson Lakes \\ Campus, South Australia, 5095.
}

Email: sherin.ahamed@mymail.unisa.edu.au

\begin{abstract}
Synthetic rainfall data has a large number of uses. Specifically, the behaviour of a reservoir under most scenarios can be best understood when evaluated using a large number of synthetic inputs. These synthetic values are all equally probable to occur but many of them may not have appeared in the historical record. Stochastic weather generators produce these synthetic data that were statistically equal to historical records. Different approaches have been used to generate synthetic rainfall data, Markov Chains being the most frequently used method. One of the major drawbacks of the current models is that when synthetically generated daily rainfall is aggregated into monthly totals, it fails to demonstrate all statistical properties of monthly series. The same could be seen in seasonal and annual series as well. The above drawback is very significant in preserving the variance of the series. Since daily rainfall is the basis of higher order synthetic rainfall series, the preservation of all statistical properties should be a pre-requisite for its application in numerous hydrological, ecological and agricultural contexts.
\end{abstract}

A disaggregation rainfall model is presented in this paper that overcomes the drawbacks of earlier models, which could not produce coinciding statistical moments at all the time scales discussed above. In this model first a seasonal value is generated; followed by monthly values to tally with the seasonal total; and finally, daily values to tally with the monthly totals. Daily, monthly and seasonal rainfall have a skewed pattern, on $[0, \infty)$. A Gamma distribution is often used in the literature to generate rainfall amounts due to its similarity to the above pattern. However, some of the values generated using the Gamma distribution are high and unrealistic as it has no upper bound. Use of Beta distribution from the same family overcomes this limitation. Upper and lower limits are found using an Extreme Value distribution. The correlation structure of the rainfall differs with the place and time. When generating synthetic rainfall both the independent and correlated cases were considered. Spearman's correlation coefficient is used to find the correlation structure in the seasonal and monthly rainfall values while a Markov Chain is used to capture the underlying correlation in the daily rainfall. The Kolmogorov- Smirnov statistical test is used to check whether the simulated and observed values have statistically indistinguishable cumulative probability distributions. The model is demonstrated using data from two locations, Hume in New South Wales and Pooraka in South Australia.

Keywords: rainfall, synthetic data generation, disaggregation, markov chain 
Ahamed et al., Generating synthetic rainfall ...

\section{INTRODUCTION}

Rainfall modelling is very useful in hydrological, agricultural and ecological applications. In reality there is only the observed set of rainfall data. In order to understand the amount of availability of water under different environmental conditions, synthetically generated rainfall data is useful, which has an equal chance of occurrence as the observed ones. Stochastic generation of synthetic rainfall sequences is often coupled with hydrological modelling (Tarpanellia et al. 2012). Stochastic models can be divided into two parts based on its output. The first type of models give the duration of the wet spell, whereas the second type give the amount of rainfall. Similarly, stochastic models can also be divided into four types based on the time frame of representing the occurrence. They are namely, annual, seasonal, monthly and daily models. The most popular means of stochastic rainfall modelling is the Markov Chain (Gabriel and Neumann 1962).

Stochastic models of daily rainfall operate in two stages. First, they establish the occurrence of a wet or dry day, and second, the amount of rainfall on a wet day (Srikanthan and McMahon 2001). The first stage models, known as Occurrence Models, are found to be using two sources. The first concept is based on Markov Chains whereas the second on Alternative Renewal Process (Wilks and Wilby 1999). Markov Chains identify the state of each day as wet or dry and develop a relationship between the state of the current day with that of preceding days. Order one Markov Chain models were introduced by Gabriel and Neumann (1962) and have been used by many researchers in daily rainfall modelling (Stern and Coe 1984). According to Jimoh and Webster (1999), First Order models are the most popular and are adequate for many locations. They found that a second or higher order model may be required for other locations. Second or higher order models for rainfall prediction came into popularity with the work of Chin (1977). The second concept used in Occurrence Models, the Alternative Renewal Process, treat wet and dry days as a sequence of alternating wet and dry spell lengths (Roldan and Woolhiser 1982).

The second stage in a rainfall model, estimating the amount of rainfall in a wet day, is carried out using different distributions. The most prominent statistical feature of daily rainfall is that it is skewed to the right; it is very common to have large number of mild and a small number of heavy rainy days in any given location (Wilks and Wilby 1999). Thus, two parametric gamma distribution (Woolhiser and Roldan 1982; Piantadosi et al. 2009), mixed exponential distribution (Woolhiser and Pegram 1979), and truncated normal distribution (Bardossy and Plate 1992) are the main distributions used in the past.

The major drawback of stochastic models of daily rainfall is that they cannot preserve the variance of monthly and annual precipitation (Woolhiser et al. 1993; Piantadosi et al. 2009). As Piantadosi et al. (2009) noted, the current models generally perform well at the time step on which they are based. For instance, daily models simulate daily characteristics better than monthly and annual characteristics, specifically the variance. To overcome this issue, we present a rainfall model that uses a top-down approach against the usual bottom-up approach of aggregating daily rainfall values as monthly totals; monthly rainfall values as seasonal totals; and seasonal values as yearly totals.

\section{DATA AND METHODS}

\subsection{Data}

Daily and monthly official rainfall records in millimetres were obtained from the Australian Bureau of Meteorology for Hume in New South Wales $\left(36.10^{\circ} \mathrm{S}, 147.03^{\circ} \mathrm{E}\right.$, Station number: 072023) from $1922-2010$ and Pooraka in South Australia $\left(34.83^{\circ} \mathrm{S}, 138.61^{\circ} \mathrm{E}\right.$, Station number: 023026$)$ from $1884-2000$ for daily values and from $1902-2000$ for monthly values. The reason for selecting these two particular sites was to represent drastically different climatic conditions, the former with a wet and the latter with dry climate. Due to missing data, 1955, 1965 and 1994 values for Pooraka were removed from the analysis. Table (1) shows the monthly rainfall summary of these two locations.

Table 1. Summary statistics of Hume and Pooraka with mean(mm) and Standard Deviation(S.D)

\begin{tabular}{|c|c|c|c|c|c|c|c|c|c|c|c|c|c|}
\hline & Months & Jan & Feb & Mar & Apr & May & June & July & Aug & Sep & Oct & Nov & Dec \\
\hline \multirow[t]{2}{*}{ Mean } & Hume & 44.94 & 41.35 & 43.26 & 51.73 & 58.94 & 67.69 & 76.59 & 76.52 & 62.18 & 69.88 & 52.26 & 49.66 \\
\hline & Pooraka & 20.34 & 18.64 & 23.61 & 42.09 & 57.95 & 65.12 & 60.42 & 56.23 & 48.13 & 43.22 & 28.27 & 25.19 \\
\hline \multirow[t]{2}{*}{ S.D } & Hume & 46.03 & 40.36 & 36.66 & 42.44 & 42.52 & 39.27 & 38.87 & 36.98 & 32.26 & 46.96 & 35.92 & 44.06 \\
\hline & Pooraka & 20.24 & 22.08 & 24.75 & 32.40 & 33.68 & 37.13 & 27.39 & 24.79 & 24.66 & 25.45 & 21.28 & 19.84 \\
\hline
\end{tabular}


Ahamed et al., Generating synthetic rainfall ...

\subsection{Methodology}

The Gamma distribution is widely used to generate synthetic rainfall due to its flexibility of fitting all patterns of rainfall from an exponential to normal distribution. This is achieved by varying two parameters, namely, shape $(\alpha)$ and scale $(\beta)$. Nevertheless, a drawback of using the Gamma distribution for synthetic generation of rainfall total is that we can obtain high values which could be unrealistic due to its range $[0, \infty)$. Use of Beta distribution which has upper and lower bounds overcomes this limitation. The Beta distribution has a probability density function(PDF),

$$
f(x)=\frac{\Gamma(\alpha+\beta)}{\Gamma(\alpha) \cdot \Gamma(\beta)} \quad \text { for } \quad 0 \leq x \leq 1 \quad \text { and } \quad \alpha, \beta>0 \quad \text { defined on the interval [0,1]. }
$$

and can be re-scaled and shifted to obtain a Beta distribution on the interval $[a, b]$ with same shape, by the transformation, $Y=a+(b-a) X$. Then the PDF will be,

$$
f(Y)=\frac{1}{B[\alpha, \beta]} \cdot \frac{(Y-a)^{\alpha-1} \cdot(b-Y)^{\beta-1}}{(b-a)^{\alpha+\beta-1}} \quad\left\{\begin{array}{l}
a \leq Y \leq b, \\
\alpha>0, \\
\beta>0 .
\end{array}\right.
$$

where

$$
B[\alpha, \beta]=\int_{0}^{1} t^{\alpha-1}(1-t)^{\beta-1} \mathrm{~d} t .
$$

The parameters can be estimated using matching moments, then for $a=0$ and $b=1$

$$
\alpha=\bar{x}\left(\frac{\bar{x}(1-\bar{x})}{s^{2}}-1\right) \quad \text { and } \quad \beta=(1-\bar{x})\left(\frac{\bar{x}(1-\bar{x})}{s^{2}}-1\right)
$$

for $a \neq 0$, and $b \neq 1$, replace

$$
\bar{x} \quad \text { by } \quad \frac{\bar{x}-a}{b-a} \text { and } s^{2} \text { by } \frac{s^{2}}{(b-a)^{2}} \text {. }
$$

Two thirds of the observed data is used to estimate the parameter values of Beta distribution. These parameters are found by the method of matching moments. Table (2) shows the parameters of Beta distribution for daily, monthly and seasonal values for Hume and Pooraka. It should be noted that $\alpha$ and $\beta$ values of daily analysis represent a common value for the whole month rather than daily values. For increased accuracy, the analysis could be extended to calculate $\alpha$ and $\beta$ values on a daily basis. Upper and lower bounds of the Beta distribution were found by Extreme Value Distribution. The Generalized Extreme Value (GEV) distribution has the following PDF,

$$
f(x)=\left\{\begin{array}{cc}
\frac{1}{\sigma} \exp \left(-(1+k z)^{-1 / k}\right)(1+k z)^{-1-1 / k} & k \neq 0 \\
\frac{1}{\sigma} \exp (-z-\exp (-z)) & k=0
\end{array}\right.
$$

where $z=(x-\mu) / \sigma$, and $k, \sigma, \mu$ are the shape, scale, and the location parameters respectively. $\sigma>0$ and $k$ and $\mu$ are real. And the range of the distribution depends on $k$,

$$
1+k \frac{(x-\mu)}{\sigma}>0 \quad \text { if } \quad k \neq 0 \quad \text { and } \quad-\infty<x<+\infty \quad \text { if } \quad k=0
$$

$k=0, \quad k>0$ and $\quad k<0$ corresponds to Gumbel, Frechet, and reversed Weibull distributions respectively.

As an example, when the upper bound of daily rainfall was found for Hume for January, Extreme Value Distribution was fitted for the maximum value of each January for the 86 years. The 99 percentile is taken as the upper bound for the Beta distribution for daily value generation of January (Katz 1999). The same 
Ahamed et al., Generating synthetic rainfall ...

Table 2. Parameter estimates for Hume and Pooraka

\begin{tabular}{|c|c|c|c|c|c|c|c|c|c|c|c|c|c|c|}
\hline \multicolumn{5}{|c|}{ Daily } & \multicolumn{5}{|c|}{ Monthly } & \multicolumn{5}{|c|}{ Seasonal } \\
\hline & \multicolumn{2}{|c|}{ Hume } & \multicolumn{2}{|c|}{ Pooraka } & & \multicolumn{2}{|c|}{ Hume } & \multicolumn{2}{|c|}{ Pooraka } & & \multicolumn{2}{|c|}{ Hume } & \multicolumn{2}{|c|}{ Pooraka } \\
\hline & $\alpha$ & $\beta$ & $\alpha$ & $\beta$ & & $\alpha$ & $\beta$ & $\alpha$ & $\beta$ & & $\alpha$ & $\beta$ & $\alpha$ & $\beta$ \\
\hline Dec & 0.052 & 2.580 & 0.040 & 2.993 & Dec & 0.580 & 1.762 & 0.905 & 2.522 & & & & & \\
\hline Feb & 0.047 & 3.938 & 0.020 & 2.189 & Feb & 0.630 & 2.496 & 0.362 & 1.587 & & & & & \\
\hline Mar & 0.055 & 2.210 & 0.034 & 2.664 & Mar & 0.708 & 2.160 & 0.534 & 2.118 & & & & & \\
\hline Apr & 0.070 & 2.768 & 0.080 & 5.056 & Apr & 0.889 & 2.144 & 0.998 & 2.525 & MAM & 1.060 & 2.170 & 2.047 & 2.948 \\
\hline Jun & 0.134 & 2.609 & 0.161 & 4.172 & Jun & 1.093 & 2.673 & 1.465 & 2.707 & & & & & \\
\hline Jul & 0.151 & 3.266 & 0.227 & 4.409 & Jul & 1.086 & 1.838 & 1.748 & 2.860 & JJA & 1.280 & 1.640 & 1.271 & 1.849 \\
\hline Aug & 0.173 & 3.699 & 0.196 & 3.382 & Aug & 2.036 & 2.932 & 2.258 & 3.769 & & & & & \\
\hline Sep & 0.129 & 3.331 & 0.136 & 3.092 & Sep & 1.294 & 1.726 & 1.375 & 3.201 & & & & & \\
\hline Oct & 0.103 & 2.781 & 0.101 & 3.037 & Oct & 1.298 & 2.661 & 1.326 & 2.481 & SON & 2.140 & 1.960 & 1.867 & 1.762 \\
\hline Nov & 0.078 & 2.708 & 0.061 & 3.144 & Nov & 0.927 & 1.943 & 0.799 & 1.525 & & & & & \\
\hline
\end{tabular}

method is repeated with minimum values, and the first percentile of the Extreme Value Distribution becomes the lower bound of the Beta distribution. Unlike the daily values, only 86 monthly and seasonal values were available. These values were considered as a population and samples were drawn with replacement; maximum and minimum of each sample were taken and the extreme value distribution is fitted for these values. Upper and lower bounds of Beta distribution for each month and season were found as before. Table (3) shows these values for Hume and Pooraka.

The remaining data were used to cross check the model. Kolmogrov-Smirnov test was used to compare the two sets of values, with a test statistic of maximum vertical distance between the CDF of observed and generated values and the null hypothesis of no difference between CDF's. One sample proportion test was used to check whether the number of dry days in both observed and simulated values were equivalent. The null hypothesis being the simulated values, have same proportion of dry days as actual values.

Table 3. Extreme values for Hume and Pooraka

\begin{tabular}{|c|c|c|c|c|c|c|c|c|c|c|c|c|}
\hline \multicolumn{3}{|c|}{ Daily } & \multicolumn{5}{|c|}{ Monthly } & \multicolumn{5}{|c|}{ Seasonal } \\
\hline & Hume & Pooraka & & \multicolumn{2}{|c|}{ Hume } & \multicolumn{2}{|c|}{ Pooraka } & & \multicolumn{2}{|c|}{ Hume } & \multicolumn{2}{|c|}{ Pooraka } \\
\hline & upper & upper & & upper & lower & upper & lower & & upper & lower & upper & lower \\
\hline Dec & 89.54 & 64.46 & Dec & 197.14 & 0.00 & 95.24 & 0.00 & & & & & \\
\hline Jan & 132.35 & 99.78 & Jan & 213.39 & 0.50 & 119.45 & 0.00 & DJF & 379.41 & 0.00 & 156.47 & 5.46 \\
\hline Feb & 91.63 & 254.93 & Feb & 201.08 & 0.00 & 99.55 & 0.00 & & & & & \\
\hline Mar & 63.17 & 81.29 & Mar & 175.40 & 0.08 & 123.92 & 0.00 & & & & & \\
\hline Apr & 97.55 & 64.00 & Apr & 175.21 & 0.00 & 146.41 & 0.00 & MAM & 402.16 & 33.00 & 289.24 & 0.00 \\
\hline May & 54.62 & 49.81 & May & 161.39 & 0.00 & 139.08 & 0.00 & & & & & \\
\hline Jun & 47.81 & 54.12 & Jun & 229.58 & 11.00 & 192.33 & 2.90 & & & & & \\
\hline Jul & 73.59 & 35.45 & Jul & 213.20 & 17.00 & 199.75 & 10.95 & JJA & 526.12 & 56.60 & 365.57 & 51.18 \\
\hline Aug & 77.89 & 32.66 & Aug & 174.54 & 0.00 & 142.47 & 0.00 & & & & & \\
\hline Sep & 52.57 & 38.52 & Sep & 134.99 & 5.40 & 194.53 & 7.13 & & & & & \\
\hline Oct & 64.19 & 60.65 & Oct & 214.64 & 1.89 & 116.34 & 0.00 & SON & 405.84 & 31.49 & 414.56 & 21.89 \\
\hline Nov & 63.31 & 55.10 & Nov & 158.60 & 0.00 & 108.67 & 0.00 & & & & & \\
\hline
\end{tabular}

\section{Proposed Model}

The disaggregation model generated a seasonal rainfall value using the parameters relevant to a season. At the monthly level, the relevant monthly totals were generated and their sum was compared with the seasonal value for compatibility and repeated until it becomes essentially the same. These iterations were performed until a large number of synthetic rainfall values were generated. At daily level, daily rainfall totals were aggregated and compared with monthly values and repeated as above until a large number of synthetic daily rainfall values were generated.

\subsection{Seasonal Model}

A random value was generated from the uniform distribution. Since its range is from [0,1], the generated value $(r)$ can be considered as a probability. If we need to generate a value for the seasonal rainfall total for summer of Hume, $F^{-1}(r)$ was taken from the Cumulative Distribution Function(CDF) of the Beta distribution that corresponds to summer. In this way 1000 rainfall totals for summer of Hume and Pooraka were generated. Figure (1) shows the CDF of the observed and generated values and they coincide reasonably well. Table (4) shows the result of the Kolmogrov-Smirnov test to be not significant. Therefore, it can be concluded that there is no difference between generated and observed seasonal rainfall totals. 
Ahamed et al., Generating synthetic rainfall ...

\subsection{Monthly models}

Unlike seasons, some months are correlated. As monthly rainfall values are skewed, only the Spearman's Correlation Coefficient can be used to find the correlation between months. Spearman's Correlation Coefficient is given by, $r_{i j}=\frac{1-6 \sum_{k=1}^{n} d_{k}^{2}}{n\left(n^{2}-1\right)}$, where $d_{k}$ is the difference between the ranked pairs and $\mathrm{n}$ is the number of pairs. Table (5) shows the correlation between the adjacent months and the corresponding $p$-values. January denotes the correlation between December and January. The null hypothesis that monthly rainfall values were uncorrelated was rejected if $p$-value $<0.05$. It can be seen that some months were correlated in Hume and almost all months were independent in Pooraka except September. Therefore, correlation was taken into consideration when monthly values were generated.

Table 4. Kolmogorov-Smirnov test results for Hume

\begin{tabular}{|l|l|l|l|l|}
\hline & Daily & \multicolumn{2}{|c|}{ Monthly } & Seasonal \\
\hline & & independent & correlated & \\
\hline Observed data points(m) & 930 & 30 & 30 & 30 \\
\hline Simulated data points(n) & 2000 & 1000 & 1000 & 1000 \\
\hline Test Statistics & 0.031 & 0.2177 & 0.1977 & 0.1753 \\
\hline Critical value at 5\% sig. level & 0.054 & 0.2520 & 0.2520 & 0.2520 \\
\hline Results of the test & not sig. & not sig. & not sig. & not sig. \\
\hline
\end{tabular}

Table 5. Spearman's rank correlation between successive months for Hume and Pooraka

\begin{tabular}{|l|l|l|l|l|}
\hline & \multicolumn{2}{|c|}{ Hume } & \multicolumn{2}{c|}{ Pooraka } \\
\hline Months & correlation & p-value & correlation & p-value \\
\hline January & 0.239 & $\mathbf{0 . 0 1 6}$ & 0.166 & 0.068 \\
February & 0.220 & $\mathbf{0 . 0 2 5}$ & 0.165 & 0.069 \\
March & 0.265 & $\mathbf{0 . 0 0 8}$ & 0.014 & 0.449 \\
April & 0.099 & 0.191 & -0.079 & 0.759 \\
May & 0.180 & 0.055 & 0.047 & 0.337 \\
June & 0.104 & 0.173 & 0.122 & 0.138 \\
July & 0.148 & 0.095 & 0.185 & $\mathbf{0 . 0 4 8}$ \\
August & 0.145 & 0.100 & 0.171 & 0.062 \\
September & 0.142 & 0.104 & 0.199 & $\mathbf{0 . 0 3 7}$ \\
October & 0.393 & $\mathbf{0 . 0 0 0}$ & 0.064 & 0.284 \\
November & 0.221 & $\mathbf{0 . 0 2 4}$ & 0.153 & 0.085 \\
December & 0.270 & $\mathbf{0 . 0 0 7}$ & 0.164 & 0.071 \\
\hline
\end{tabular}

Independent monthly models. Independent months were generated as the seasonal values. Figure (3) shows that the observed and generated values coincide reasonably well for July of Hume. Table (4) shows the same result when the K-S test was used.

Correlated monthly models. For correlated monthly rainfall, the method followed by Zakaria (2011)was used. This method is restricted to common shape $(\alpha)$ and different scale $\left(\beta_{i}\right)$ parameters. By considering $n$ correlated gamma variables $\left\{X_{i}\right\}_{i=1}^{n}$ with parameters $\alpha$ and $\beta_{i}$ where the PDF of $\left\{X_{i}\right\}_{i=1}^{n}$ and the PDF of $Y=\sum_{i=1}^{n} X_{i}$ of the sum of $n$ correlated gamma variables were given by,

$f_{i}\left(x_{i} ; \alpha, \beta\right)=\frac{x_{i}^{\alpha-1} e^{-x_{i} / \beta_{i}}}{\Gamma(\alpha) \beta_{i}^{\alpha}} \quad$ and $\quad f_{Y}(y)=\prod_{i=1}^{n}\left(\frac{\lambda_{1}}{\lambda_{i}}\right)^{\alpha} \sum_{k=0}^{\infty} \frac{\delta_{k} y^{n \alpha+k-1} \exp \left(-y / \lambda_{1}\right)}{\lambda_{1}^{n \alpha+k} \Gamma(n \alpha+k)} \quad$ respectively.

Here the $\left\{\lambda_{i}\right\}_{i=1}^{n}$ is the eigen values of the matrix $\mathrm{A}=\mathrm{DC}$ and $\lambda_{1}$ is the minimum eigen value and $\mathrm{A}$ is assumed to be positive definite.

The Matrices, $\quad D=\left[\begin{array}{cccc}\beta_{1} & 0 & \ldots & 0 \\ 0 & \beta_{2} & \ldots & 0 \\ \vdots & \vdots & \ddots & \vdots \\ 0 & 0 & \ldots & \beta_{n}\end{array}\right]_{n \times n} \quad C=\left[\begin{array}{cccc}1 & \sqrt{\rho_{12}} & \ldots & \sqrt{\rho_{1 n}} \\ \sqrt{\rho_{21}} & 1 & \cdots & \sqrt{\rho_{2 n}} \\ \vdots & \vdots & \ddots & \vdots \\ \sqrt{\rho_{n 1}} & \sqrt{\rho_{n 2}} & \ldots & 1\end{array}\right]_{n \times n}$ where the $\rho_{i j}$ is the Spearman's correlation coefficient.

And $\delta_{k}$ is given by, $\quad \delta_{k+1}=\frac{\alpha}{k+1} \sum_{i=1}^{k+1}\left[\sum_{j=1}^{n}\left(1-\frac{\lambda_{1}}{\lambda_{j}}\right)^{i}\right] \delta_{k+1-i} \quad$ for $k=0,1,2, \ldots$ where $\delta_{0}=1$.

For the correlated months the common shape $\bar{\alpha}=\left(\alpha_{1}+\alpha_{2}+\ldots . .+\alpha_{n}\right) / n \quad$, and the corresponding $\beta_{i}$ 's of the months were recalculated by the method of matching moments, $\alpha \beta=\bar{x}$ and $\alpha \beta^{2}=s^{2}$. 
Ahamed et al., Generating synthetic rainfall ...

Using this method rainfall totals for correlated months were generated using Gamma distribution instead of Beta distribution, since this method cannot be extended to Beta distribution.

The monthly values generated in this way seems to tally well with the observed values as shown in Figure (4) for January of Hume. K-S test presented in Table (4) gives the same result. Table (6) is used here to demonstrate the standard deviation of Hume for January obtained from the model closely resembles that of the observed.

Table 6. Standard Deviation of observed and generated values for January, Hume.

\subsection{Daily models}

\begin{tabular}{ccc}
\hline & Observed values & generated values \\
\hline Hume & 46.03 & 45.00 \\
\hline
\end{tabular}

A day can be either dry or wet and it depends on the preceeding day. The underlying correlation structure in the daily rainfall can be captured by the Markov Chain and the status of the day can be represented by a two state Markov Chain. Then the transition probability could be given by,

$p_{i j}=P($ a day changing status from $i$ to $j)=\frac{P\left(X_{t+1} \in j \mid X_{t} \in i\right)}{P\left(X_{t} \in i\right)}=\frac{\text { Frequency of } X_{t} \in i \rightarrow X_{t+1} \in j}{\text { Frequency of } X_{t} \in i}$

where $i, j \in\{$ dry,wet $\}$. The transition probability matrix (t.p.m) gives the probability of movement from one state to another. The t.p.m P, and the corresponding Equilibrium vector V for January are given by,

$$
\mathbf{P}=\left(\begin{array}{ll}
0.87 & 0.13 \\
0.61 & 0.39
\end{array}\right) \quad \text { and } \quad \mathrm{V}=(0.17298,0.82702) \quad \text { respectively. }
$$

The vector $V$ is found either by raising $P$ to a higher power until it will have the same probabilities in all rows or by solving the equations, $V P=V$ and $v_{1}+v_{2}=1$.

In daily rainfall generation, first a random value was generated from the uniform distribution. Using the equilibrium vector, the status of the day was decided. If it is wet, a synthetic daily rainfall total was generated using similar method as for seasonal and monthly generation from the Beta distribution with the corresponding $\alpha$ and $\beta$. This would be the rainfall total for the first day of the month. For the second day, a random value was generated from Uniform distribution and using the transition probability matrix the status of the day was found. Like earlier, if it is wet, the rainfall total was generated. This method is repeated for the rest of the month. Figure(2) shows the CDF's of observed and generated values of January for Hume with a good fit. The K-S test shown in Table (4) gives the same result. The results of the one sample proportion test shown in Table(7) indicates the proportion of dry days to be equivalent in both observed and simulated values.

Table 7. Trial outcomes of 1-sample proportion test for no. of dry days of January, Hume.

\begin{tabular}{cccc}
\hline Observed proportion & Simulated proportion & exact $p$-value & Results of the test \\
\hline 0.827 & $1686 / 2000$ & 0.06 & not sig. \\
\hline
\end{tabular}

\section{Conclusion}

The proposed disaggregation model performed well for Hume and Pooraka which are wet and dry locations in NSW and SA respectively. This model could be used to accurately predict synthetic rainfall for South Australia. Synthetic rainfall totals are used as inputs in many applications particularly simulation of run-off from a watershed, moisture budgeting, crop planning, scheduling of irrigation, flood hazard mapping and design of hydraulic structures with the increasing importance given to climate change predictions. All above applications need accurate and long term rainfall data which could only be met with synthetic data. As future work, inter-annual variability for the aggregated series would be examined in detail.

\section{REFERENCES}

Bardossy, A. and E. Plate (1992). Space time model for daily rainfall using atmospheric circulation patterns. Water Resour. Res. 28, 1247-1259.

Chin, E. (1977). Modelling daily precipitation occurrences process with markov chain. Water Resour. Res. 13, 949-956.

Gabriel, K. and J. Neumann (1962). A markov chain model for daily rainfall occurrence at tel aviv. Q. J.R. Meteorol. Soc. 88, 90-95. 
Ahamed et al., Generating synthetic rainfall ...

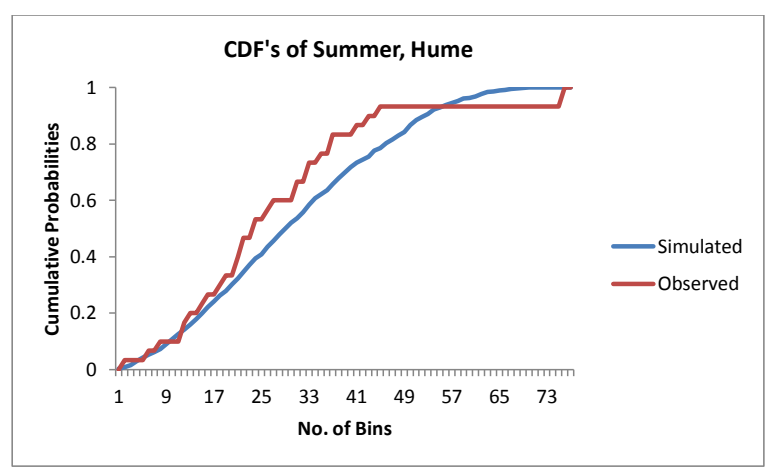

Figure 1. CDF's of Summer,Hume.

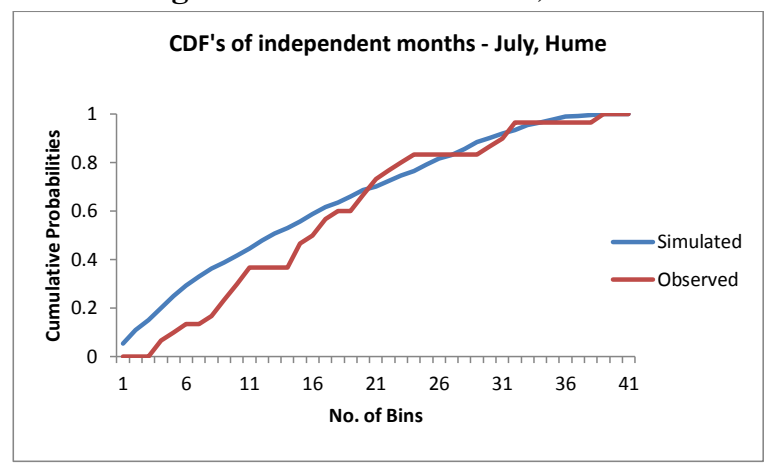

Figure 3. CDF's of independent months-July, Hume.

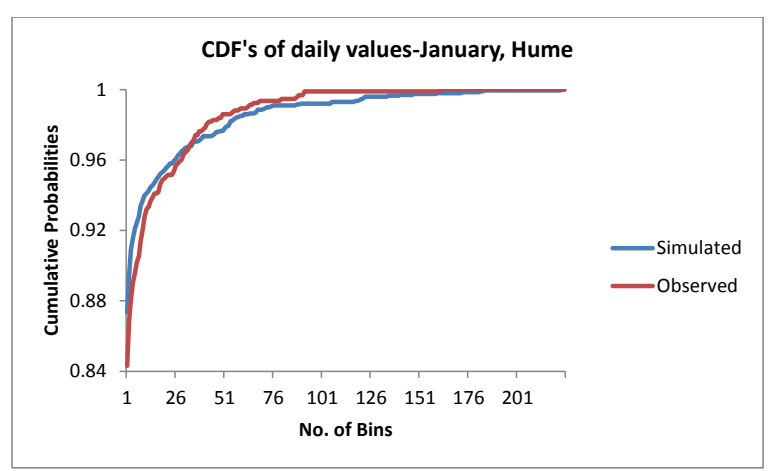

Figure 2. CDF's of Daily values-January, Hume

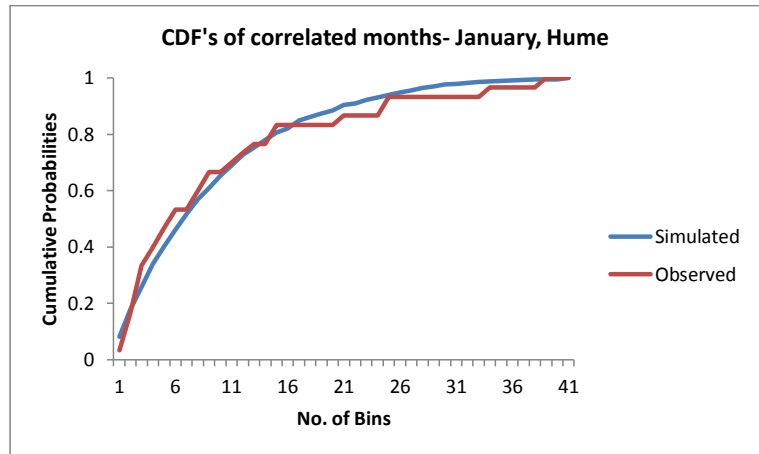

Figure 4. CDF's of correlated months-January, Hume

Jimoh, O. and P. Webster (1999). Stochastic modelling of daily rainfall in nigeria, intra-annual variation of model parameters. J. Hydrol. 222, 1-17.

Katz, R. (1999). Extreme value theory for precipitation: sensitivity analysis for climate change. Adv. Water Resour. 23, 133-139.

Piantadosi, J., J. Boland, and P. Howlet (2009). Generating synthetic rainfall on various timescales - daily, monthly and yearly. Environ Model Assess 14, 431-438.

Roldan, J. and D. Woolhiser (1982). Stochastic daily precipitation models 1.a comparison of occurrence processes. Water Resour. Res. 18(5), 1451-1459.

Srikanthan, R. and T. A. McMahon (2001). Stochastic generation of annual, monthly and daily climate data: A review. Hydrology and Earth System Sciences 5(4), 653-670.

Stern, R. and R. Coe (1984). A model fitting analysis of daily rainfall data. J. R. Stat. Soc. A. 147, 1-34.

Tarpanellia, A., M. Franchinib, L. Broccaa, S. Camicia, F. Melonea, and T. Moramarcoa (2012). A simple approach for stochastic generation of spatial rainfall patterns. J. Hydrol. 472(23), 63-76.

Wilks, D. and R. Wilby (1999). The weather generation game: a review of stochastic weather models. Progress in Physical Geography 23(3), 329-357.

Woolhiser, D., T. Keefer, and K. Redmond (1993). Southern oscillation effects on daily precipitation in the south-western united states. Water Resour. Res. 29, 1287-1295.

Woolhiser, D. and G. Pegram (1979). Maximum likelihood estimation of fourier coefficients to describe seasonal variation of parameters in stochastic daily precipitation models. J. Appl. Meteorol. 18, 34-42.

Woolhiser, D. and J. Roldan (1982). Stochastic daily precipitation models 2.a comparison of distributions of amounts. Water Resour. Res. 18(5), 1461-1468.

Zakaria, R. (2011). Mathematical modelling of rainfall in the murray darling basin. Ph.D. Thesis, University of South Australia. 